\title{
DYNAMICS OF A BANDPASS SIGMA-DELTA MODULATOR AS A PIECEWISE ISOMETRY
}

\author{
P. Ashwin, J.H.B. Deane and X-C Fü \\ School of Electronic Engineering, IT and Mathematics, \\ University of Surrey, \\ Guildford, \\ GU2 7XH, UK, \\ e-mail: P.Ashwin/Fu-Xin-Chu@ex.ac.uk, J.Deane@eim.surrey.ac.uk
}

\begin{abstract}
We examine a model for a bandpass $\Sigma \Delta$ modulator introduced by Feely and co-workers. This is shown to have the dynamics of a piecewise isometry of a union of convex polygons on the plane by an appropriate transformation of the linearised parts into normal form. Using these we show that the periodically coded regions form a packing of the phase space by circles and we link this system to a number of similar ones. In particular we conjecture that for typical values of the parameter $\theta$ there is a positive measure set of points that have aperiodic codings.
\end{abstract}

\section{INTRODUCTION}

We consider the iterated mapping

$$
\begin{aligned}
u_{n+2}= & 2 r \cos \theta w_{n+1}-r^{2} w_{n} \\
& +2 r \cos \theta\left(x_{n+1}-\operatorname{sgn} w_{n+1}\right) \\
& -r^{2}\left(x_{n}-\operatorname{sgn} w_{n}\right)
\end{aligned}
$$

used, in Feely and Fitzgerald [2], as a model for the bandpass $\Sigma \Delta$ modulator shown in figure 1 .

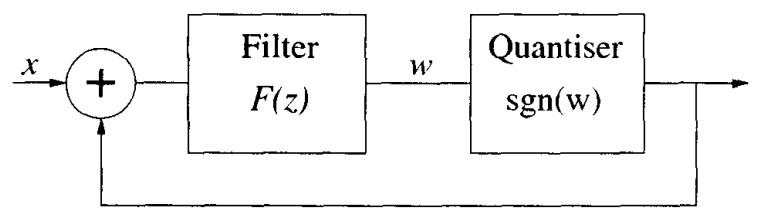

Figure 1: A block diagram of the $\Sigma \Delta$ modulator considered in this paper.

The feedback loop of the modulator contains a filter with transfer function

$$
F(z)=\frac{2 z \cos \theta-1}{z^{2}-2 z \cos \theta+1}
$$

* Present address: School of Mathematical Sciences, Laver Building. University of Exeter. Exeter EX4 4QE. UK.

${ }^{\dagger}$ Work supported by the EPSRC on grant number GR/M36335 where $\theta$ is a parameter that sets the centre frequency of the passband. The output from the modulator is the quantisation $\operatorname{sgn}\left(w_{n}\right)$ given the input $x_{n}$. We are interested in the quiescent behaviour, $x_{n} \equiv 0$, with unity gain, $r=1$, and so we re-write the mapping in vector form as

$$
\begin{aligned}
\left(\begin{array}{c}
w_{n+1} \\
t_{n+1}
\end{array}\right)= & \left(\begin{array}{cc}
0 & 1 \\
-1 & 2 \cos \theta
\end{array}\right)\left(\begin{array}{c}
w_{n} \\
t_{n}
\end{array}\right) \\
& +\left(\begin{array}{c}
\operatorname{sgn} w_{n}-2 \cos \theta \operatorname{sgn} t_{n}
\end{array}\right)
\end{aligned}
$$

The eigenvalues of the matrix are $\mathrm{e}^{ \pm i \theta}$. The first part of the transformation (2) therefore preserves area and so can be conjugated to a solid rotation by shearing the coordinates. In this paper we show that the mapping (2) is invertible on a set $M$, and displays a globally attracting set, also $M$, whose geometry we determine. The consequences of this for the dynamics of the $\Sigma \Delta$ modulator are then discussed.

\section{REDUCTION TO INVERTIBLE PIECEWISE ISOMETRY ON TWO TRAPEZIA}

We now define $u_{n}=w_{n} / \sin \theta-t_{n} \cot \theta, v_{n}=t_{n}$, and the state vector $\mathbf{x}_{n}=\left[u_{n}, v_{n}\right]^{T}$. With this transformation (2) can be written in Jordan normal form as

$$
\mathbf{x}_{n+1}=f\left(\mathbf{x}_{n}\right)=R \mathbf{x}_{n}+\sigma\left(\mathbf{x}_{n}\right)\left(\begin{array}{c}
-\cot \theta \\
1
\end{array}\right)
$$

where $\sigma\left(\mathbf{x}_{n}\right)=\operatorname{sgn}\left(u_{n} \sin \theta+v_{n} \cos \theta\right)-2 \cos \theta \operatorname{sgn} v_{n}$ and $R$ represents a pure clockwise rotation by $\theta$. With the mapping in this form, the $u-v$ plane is split into the four infinite triangular sectors $A-D$ shown in figure 2, each with a different value of $\sigma$. From this figure and equation (3), it is possible to deduce the effect of one iteration of the mapping. The result for all four sectors is shown in figure 3 . We have used $\theta=1.8$ radians for drawing figures 2-6, which illustrates the generic behaviour that is observed for 
$\pi / 3 \leq \theta \leq 2 \pi / 3$ and $4 \pi / 3 \leq \theta \leq 5 \pi / 3$. Note that the region bounded by $B_{1}^{\prime} O B^{\prime} O A^{\prime} A_{2}^{\prime}$ contains points from both sectors $A$ and $B$, and similarly, parts of both $C$ and $D$ are mapped into $C_{2}^{\prime} O C^{\prime} O D^{\prime} D_{1}^{\prime}$.

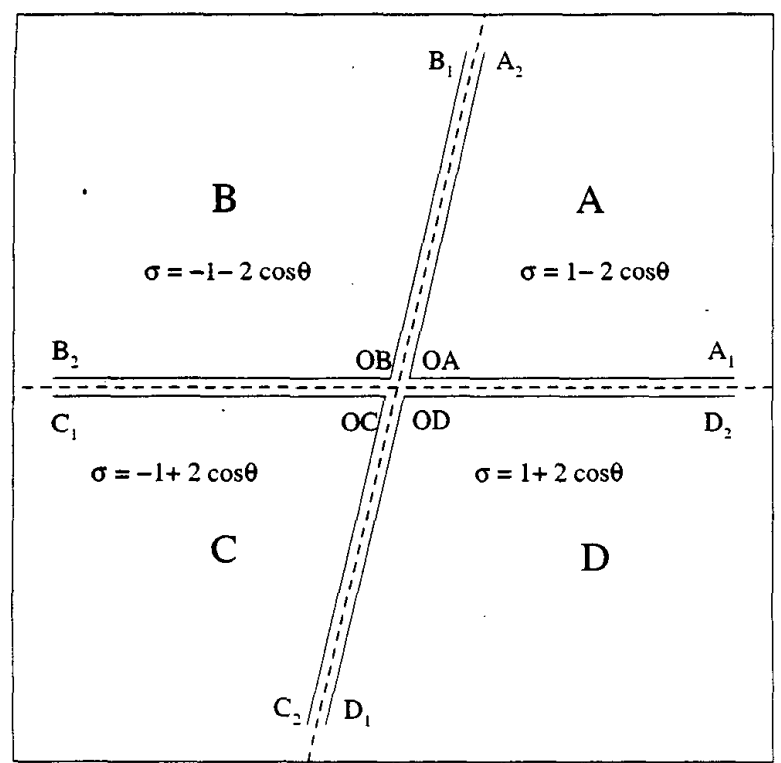

Figure 2: The $u-v$ plane is split into four infinite triangular sectors by the lines of discontinuity (shown dashed) of the mapping (3). The obtuse angle between the dashed lines is $\theta$.

The result of applying the mapping a second time, to regions $U E O A^{\prime} A_{2}^{\prime}$ and $B_{1}^{\prime} O B^{\prime} F V$ of figure 3 , is shown in figure 4 . (The effect on the corresponding sectors on the left hand side of figure 3 can be seen by rotating figure 4 by $180^{\circ}$.)

Hence the mapping can be reduced to a rearrangement of the four kite-shaped quadrilaterals $P \equiv H G O A^{\prime \prime} E^{\prime}$, $Q \equiv J F^{\prime} O B^{\prime \prime} K^{\prime}$ and $R . S$ symmetrically defined, ${ }^{l}$ shown in figure 5 , in such a way that

$$
M=P \cup Q \cup R \cup S=f(P) \cup f(Q) \cup f(R) \cup f(S)
$$

apart from a set of zero measure. A result in [1] implies that if this holds for an area preserving map $f$, then $f$ must be invertible on $M$ except possibly on a set of zero measure.

Looking at figure 3 , we can see that there exist values of $\theta$ such that the maximal invariant subset $M$ is a global attractor for all initial conditions in the $u-v$ plane, with the possible exception of those that lie on the lines of discontinuity. For instance, the points $O A^{\prime}$ and $O B^{\prime}$ in figure 3 , have co-ordinates $\left(\cot \theta(-1+2 \cos \theta)^{+}, 1-2 \cos \theta\right)$ and

\footnotetext{
${ }^{1} P$ is $\left(0^{+} \cdot 0^{+}\right),\left(\csc \theta \cdot 0^{+}\right),(\csc \theta(1+\cos 3 \theta),-1-2 \cos 2 \theta)$, $\left(-\cot \theta(1-2 \cos \theta)^{+} .1-2 \cos \theta\right)$ and $Q$ is $\left(0^{-} .0^{+}\right),(-\cot \theta(1+$ $\left.2 \cos \theta)^{-}, 1+2 \cos \theta\right) \cdot(\csc \theta(\cos 3 \theta-1),-1-2 \cos 2 \theta) \cdot\left(-\csc \theta \cdot 0^{+}\right)$.
}

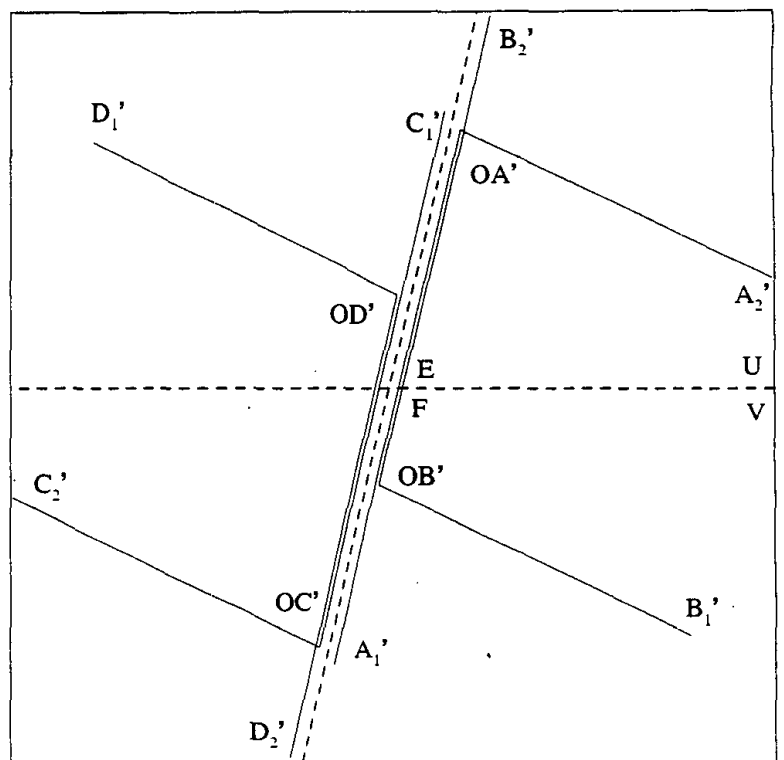

Figure 3: The effect of the mapping on the sectors of figure 2. Primed points are mapped from figure 2.

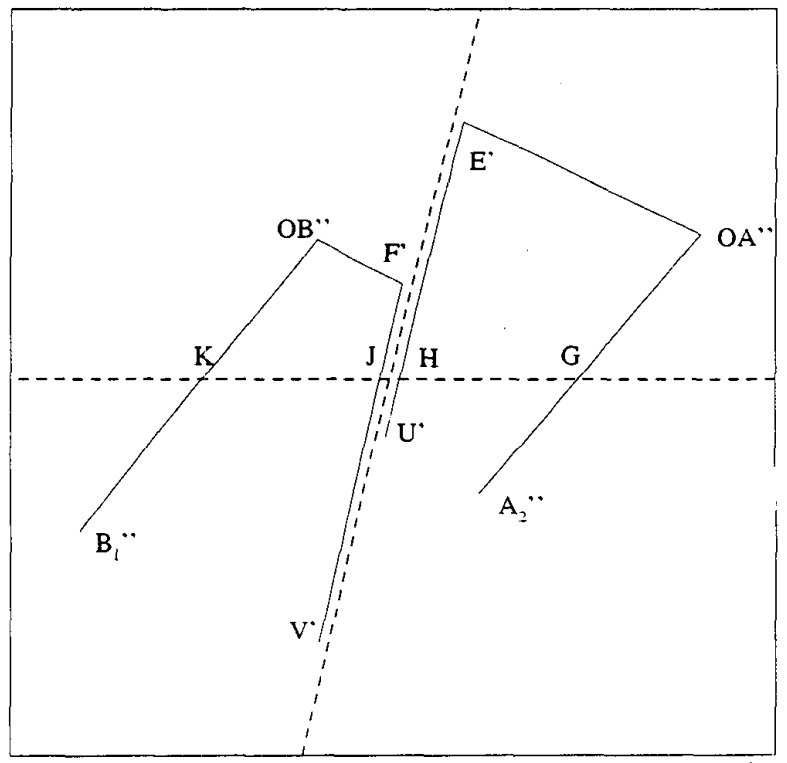

Figure 4: The effect of the second iterate of the mapping. 

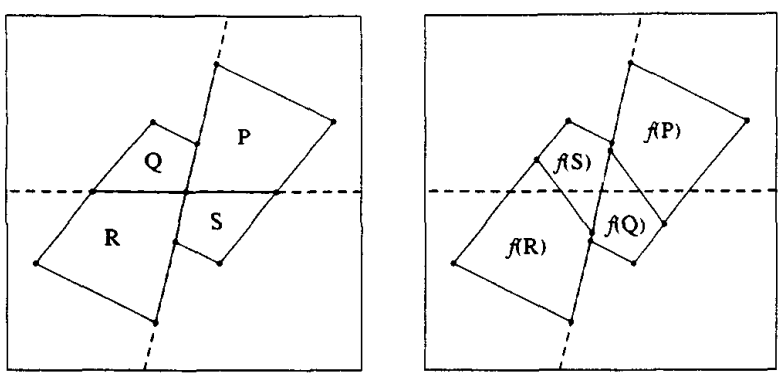

Figure 5: Left: the set $M=P \cup Q \cup R \cup S$ on which the mapping $f$ is invertible; right: the effect of $f$ on each of $P$, $Q, R$ and $S$ leaves their union $M$ unchanged. Hence, $f$ is invertible on $M$.

$\left(\cot \theta(1+2 \cos \theta)^{+},-1-2 \cos \theta\right)$. These points lie above and below the $u$-axis respectively for $\theta=1.8$, the value used to draw figures 2-6. Hence, every iteration of $f$ will move points not already in $M$ towards $M$.

This mapping is one of the simplest possible piecewise isometries that can have arbitrary rotations and the dynamics can be constructed simply by considering the action of the map on one of the trapezia, $P \cup S$ or $Q \cup R$; see [1].

\section{CODINGS OF TRAJECTORIES}

It is clearly possible to associate the state of this system with one of the four possible states of the quantising circuit over two consecutive timesteps. This corresponds to classifying the state $s_{i}$ of the system by $\{P, Q, R, S\}$ in figure 5 and leads to a coding of a trajectory, starting from a given initial condition $\mathbf{x}_{0}$, by a semi-infinite string of the form

$$
\mathbf{x}_{0} \leftrightarrow s_{0} s_{1} s_{2} \cdots
$$

where $s_{k}=P$ if $f^{k}\left(\mathbf{x}_{0}\right) \in P, s_{k}=Q$ if $f^{k}\left(\mathbf{x}_{0}\right) \in Q$ etc.

We can use the fact that the mapping reduces to an invertible piecewise isometry on the convex regions $P, Q, R, S$ to infer much about the dynamics of the mapping. Given a string of symbols $s_{0} s_{1} s_{2} \ldots$ we say this is periodic if it repeats after a fixed number of symbols. Theorems of Ashwin [1] and Goetz [3] imply that the set of periodically coded points of such maps takes the form of a disjoint union of convex regions that are discs if the rotations $\theta / \pi$ are irrational, and polygons otherwise. The largest among these discs are visible in figure 6. The largest disc in $P$ and in $R$ corresponds to the coding $P P P \ldots$ and $R R R \ldots$ respectively; the centres of these discs are $( \pm(1-2 \cos \theta) / 2 \sin \theta$, $\pm(1-2 \cos \theta) / 2(1-\cos \theta))$ and are fixed points of $f$ of period one. The largest discs in $Q$ and $S$ have coding sequences $Q S Q S \ldots$ and $S Q S Q \ldots$ respectively, and their centres are period-two fixed points of $f$. Within the largest

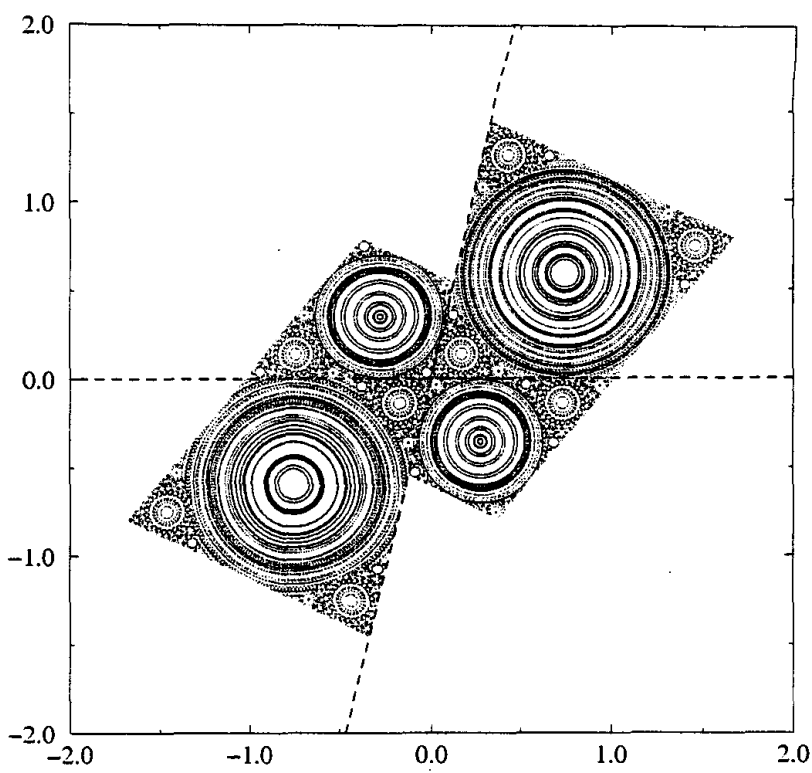

Figure 6: An illustration of the dynamics of iterates of $f$ when $\theta=1.8$. The diagram was generated by choosing 150 random initial conditions within $M$ and plotting 500 iterates, starting from each of these initial points.

discs in $P$ and $R$, the dynamics is the same as for the circle mapping with angle of rotation $\theta$.

There are a number of difficult open questions related to this dynamically defined invariant disc packing. First, are there an infinite number of periodic orbits? Second, is there any measure in the complement of this periodic packing? Numerical results suggest that the answer to both of these is positive but as yet there is no proof. A search with $\theta=1.8$ revealed codings of periods $1(\times 2), 2,8,12(\times 2), 14,50$, $57,64,82,106(\times 2), 294,300,313,330,745,794,900 \ldots$ The total area of discs with codings of these periods is approximately $89 \%$ of the area of $M$. If the second question were to be true - as the numerical work suggests - this would imply a non-zero probability of observing aperiodic behaviour in this system.

All the foregoing is valid for $\pi / 3<\theta<2 \pi / 3$ and $4 \pi / 3 \leq \theta \leq 5 \pi / 3$ and figures $2-6$ illustrate the generic case. For $\theta=\pi / 3$ and $5 \pi / 3$, kites $P$ and $R$ degenerate into lines, and $Q$ and $S$ become equilateral triangles of side $4 / \sqrt{3}$. For $\theta=2 \pi / 3$ and $4 \pi / 3, P, R$ and $Q . S$ swap roles. These can be deduced from the co-ordinates of the vertices of $P . Q, R, S$ given in footnote 1 . All points within $M$ in these cases are of course periodic points, since $\theta / \pi$ is rational.

For $\theta$ outside the range considered above, all we can say at present is that the geometry of $M$ can be radically different - see figure $7(\theta=0.65)$ for an example. 


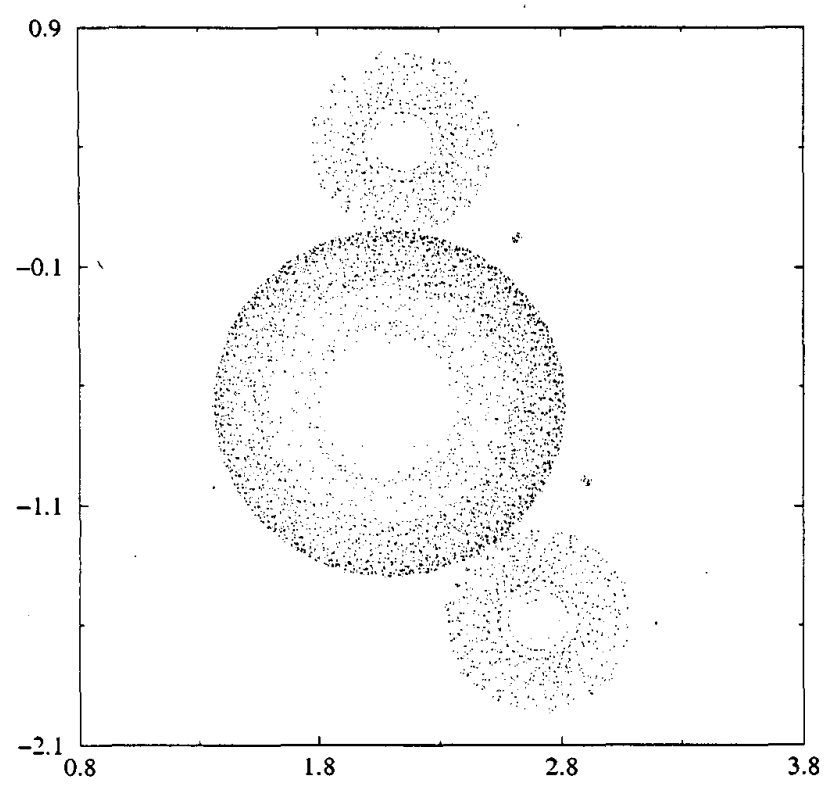

Figure 7: One half of the attracting set $M$ for $\theta=0.65$, showing very different behaviour from the other cases considered in this paper. The other half of the set is the above but with $\left(u \cdot v^{\cdot}\right) \rightarrow(-u .-v)$.

Note that this map is very close in form to the following map of the plane studied in detail by Goetz [3]. We define an iteration on $z \in \mathbb{C}$ by $z_{n+1}=e^{i \theta}\left(z_{n}-u\left(z_{n}\right)\right)$ where

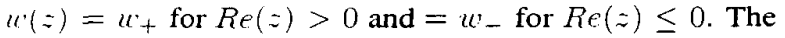
map discussed here has two discontinuities rather than the one discontinuity on $\operatorname{Re}(z)=0$ of Goetz map. However. recent work of Mendes [5] demonstrates that there can be polygonal invariant attractors for the Goetz map in certain parameter regimes.

\section{CONCLUSIONS}

We have shown that, for a certain range of the parameter $\theta$, the mapping $f$ that describes the behaviour of the bandpass $\Sigma \Delta$ modulator is a piecewise isometry after a coordinate change. This allows us to show that it is (a) invertible on a polygonal subset $M$, which we have determined; (b) the map possesses a global attractor, $M ;$ (c) there is an invariant disc packing of $M$ composed of periodic and quasiperiodic solutions if $\theta / \pi$ is irrational and periodic solutions otherwise: (d) the complement of this invariant disc packing (which may have positive measure) possesses orbits whose codings are all aperiodic and may display complicated dynamical behaviour. Recent results [1] indicate that the invariant disk packing has no tangencies and in this sense is a 'loose' packing.

All the above is valid in the special case of zero input $\left(x_{n}=0\right.$ for all $\left.n\right)$ and unity gain $(r=1)$ and it is likely to be much more difficult to prove rigorously any such results in other cases. However, we believe that this special case qualitatively illustrates some aspects of the behaviour that can be expected for $r$ close to unity.

We conjecture that there can exist mixing solutions for the system in the sense that a set of initial conditions of positive measure shows sensitive dependence. By seeing both as examples of piecewise isometries we have made a rigorous connection between this system and the overflow oscillation problem (see for example [4]) for digital filters.

\section{REFERENCES}

[1] P. Ashwin and X-C. Fu, On the geometry of planar piecewise isometries (in preparation, 2001); P. Ashwin and X-C. Fu, Tangencies in invariant disk packings for certain planar piecewise isometries are rare (submitted 2001); P. Ashwin, Elliptic behaviour in the sawtooth standard map, Physics Letters A, 232, p. 409 (1997); P. Ashwin, X-C. Fu and J. Terry, Riddling of invariant sets for some discontinuous maps preserving Lebesgue measure, (submitted, 2000).

[2] O. Feely and D. Fitzgerald, Non-ideal and chaotic behaviour in bandpass sigma-delta modulators Proceedings of NDES 1996, Sevilla, Spain, pp. 399-4.04 (1996); O. Feely, Nonlinear dynamics of bandpass sigma-delta modulation, Proceedings of NDES, Dublin, pp 33-36 (1995); O. Feely and D. Fitzgerald, Bandpass sigma-delta modulation: an analysis from the perspective of nonlinear dynamics, Proceedings of ISCAS 1996, Atlanta, Ga, May 1996. A.C. Davies, Periodic Non-linear Oscillations from Bandpass sigmadelta modulators', Proceedings of ISCAS 1996, Atlanta, Ga, pp. 469-472 (1996).

[3] A. Goetz, Dynamics of piecewise isometries, Illinois Joumal of Mathematics, 44 p. 465 (2000); A. Goetz, Perturbation of 8-attractors and births of satellite systems, International Journal of Bifurcation \& Chaos, 8 p. 1937 (1998).

[4] L. Kocarev, C.W. Wu and L.O. Chua, Complex behaviour in Digital filters with overflow nonlinearity: analytical results. IEEE Trans CAS-II 43, pp. 234-246 (1996); A.C. Davies, Nonlinear oscillations and chaos from digital filter overflow. Philosophical Transactions of the Royal Society of London A, 353, pp. 85-99 (1995)

[5] M. Mendes, University of Surrey preprint (in preparation, 2000). 